fasciculations ceased. On the 11th day, he developed severe stridor, bilateral vocal cord paralysis, and areflexia with proximal neuropathy. By day 27 , deep tendon reflexes had returned, and EMG and NCS were normal. (Aiuto LA et al. Life-threatening organophosphate-induced delayed polyneuropathy in a child after accidental chlorpyrifos ingestion. I Pediatr April 1993; 122: 658-660). (Reprints: Leslie A Aiuto MD, Div of Pediatric Critical Care Medicine, Dept of Pediatrics, North Shore University Hospital, 300 Community Dr, Manhasset, NY 11030).

COMMENT. Organophosphate-induced delayed polyneuropathy has been described previously only in adults. Clinical manifestations begin 1 to 3 weeks after the acute cholinergic crisis. Signs of a distal, symmetric, motor polyneuropathy may be complicated by pyramidal tract and CNS involvement. Residual deficits are common.

\title{
IPECAC-INDUCED NEUROMUSCULAR WEAKNESS
}

A 15-month-old girl was admitted to the University of Maryland, Department of Pediatrics, with recurrent vomiting after two previous hospitalizations for vomiting, proximal weakness, and ataxia. Weakness and hypotonia progressed, she could not sit, and deep tendon reflexes were depressed. She was hypotensive, the heart was enlarged, and the pulse rapid. Liver enzymes were markedly elevated. Muscle biopsy was consistent with a myopathy of undetermined cause. By day 12, the endotracheal tube was removed, and by day 14 she could walk. The mother had discovered an empty bottle of ipecac in the infant's toy box some 2 weeks before the initial hospitalization and 4 weeks before the onset of weakness. (Carraccio $C$ et al. Sudden onset of profound weakness in a toddler. I Pediatr April 1993; 122: 663667). (Reprints: Carol Carraccio MD, Dept of Pediatrics, Room N5W56, University of Maryland, 22 S Greene St, Baltimore, MD 21201).

COMMENT. The ipecac poisoning in this case was accidental but is sometimes intentional and a chemical method of child abuse. Emetine produces a neuromuscular weakness of moderate to severe degree by a direct toxic effect on muscle. Cardiomyopathy is an additional side effect of emetine overdose.

\section{INFECTIOUS DISORDERS}

\section{HIV NEUROLOGIC COMPLICATIONS AND HEMOPHILIA}

A multicenter prospective study of the growth and neuropsychological function of 207 boys (mean age 12 years) with hemophilia and seropositive for human immunodeficiency virus type 1 (HIV-1) is reported from the University of Iowa College of Medicine, Iowa City. Head trauma was sustained in $40 \%$, intracranial hemorrhage occurred in $10 \%$, and seizures in $9 \%$. 
Behavioral disorders in $15 \%$ and intracranial infections in $4 \%$ of the total were most frequent in the HIV positive group. Neurologic abnormalities, involving cranial nerves (11\%), deep tendon reflexes $(17 \%)$, coordination $(25 \%)$, muscle strength (23\%), and tone (31\%), were more common in children of 12 years and older. They were similar in frequency in HIV-seronegative and seropositive subjects, except for deep tendon reflexes and muscle tone and bulk, which were more frequently abnormal in seropositive individuals. Substantial neurological dysfunction could be attributed to hemophilia, but HIV-1 contributes to the neurologic morbidity of hemophiliacs. (Bale JF Jr et al. Neurologic history and examination results and their relationship to human immunodeficiency virus type 1 serostatus in hemophilic subjects: Results from the hemophilia growth and development study. Pediatrics April 1993; 91: 736741). (Reprints: James F Bale Jr MD, Division of Pediatric Neurology, Dept of Pediatrics, University of Iowa Hospitals and Clinica, Iowa City, IA 52242).

COMMENT. The effect of HIV-1 or its treatment on muscle and peripheral nerve was reflected by non-hemophilia-related reductions in muscle tone and bulk, identified only in HIV-1 seropositive subjects. HIV-1 was also responsible for the majority of behavioral disorders and intracranial infections observed in hemophilic children.

Of multiple MRI abnormalities, including arachnoid cysts, focal lesions, and multifocal white-matter lesions, found in 80 of 310 hemophilic boys, only diffuse cerebral atrophy was associated with HIV infection, and only in subjects with compromised immunologic function. (Mitchell WG et al. Pediatrics April 1993; 91: 742-746). Cerebral atrophy is the most consistent neuroradiological abnormality among children with HIV infection, reported in 85\%.(Mintz M, Epstein LG. Neurologic manifestations of pediatric acquired immunodeficiency syndrome: Clinical features and therapeutic approaches. Seminars in Neurology March 1992; 12: 51-56).

\section{PSYCHOLOGICAL SYMPTOMS OF SYDENHAM'S CHOREA}

The psychological symptoms of 11 children with Sydenham's chorea ( 8 girls and 3 boys, mean age 8.4 years) were evaluated at the National Institute of Mental Health, Bethesda, MD. Subjects had a recent onset of chorea and rheumatic fever. Seven had rheumatic carditis and 3 had arthritis. All were receiving penicillin, and 8 had been treated with haloperidol, without lasting benefit. All had an elevated antistreptolysin O (ASO) titer, greater than 480 Todd units, and 10 had positive antineuronal antibody titers. Psychological symptoms included nightmares, decreased attention, hyperactivity, distractibility, emotional lability, and an acute onset of obsessive-compulsive disorder. The OCD started shortly before the onset of involuntary movements, 